\title{
Limbic encephalitis occurring in association with Alzheimer's disease
}

\author{
R C Sutton, M H Lipper, H R Brashear
}

\begin{abstract}
Paraneoplastic limbic encephalitis is a rare cause of subacute dementia. A patient with limbic encephalitis and small-cell lung carcinoma is reported in whom the onset of subacute cognitive impairment was obscured by concurrent Alzheimer's disease. MRI revealed increased T2 signal in medial temporal lobes which corresponded to inflammatory pathology demonstrated at necropsy. High titres of antineuronal antibody (type II, anti-Hu) were present at death. Direct immunofluorescent staining of necropsy tissue revealed IgG bound to most remaining neurons in the temporal lobe. Antineuronal antibody screening and MRI are important in detecting limbic encephalitis.
\end{abstract}

(F Niurol Niurosurg Psichiatry 1993;56:808-811)

Limbic encephalitis was first described as a unique clinical and pathological syndrome by Brierly in $1960 .{ }^{1}$ Its association with carcinoma was elucidated by Corsellis in $1968 .^{2}$ Subsequent reports have established limbic encephalitis as one of several classic clinicopathological presentations of paraneoplastic disorders. ${ }^{3}$ Limbic encephalitis is characterised clinically by subacute cognitive deterioration with variable degrees of anxiety, confusion and memory impairment which may be difficult to differentiate from other causes of dementia. Laboratory and radiographic findings are usually non-specific. An exception is the finding of circulating antineuronal antibodies which point strongly to an underlying neoplasm. ${ }^{45}$ Since paraneoplastic limbic encephalitis and the associated malignancy are potentially treatable, it is important to differentiate this syndrome from other causes of cognitive impairment. We report the clinical, immunological, radiographic and pathological features of a case of limbic encephalitis occurring in association with Alzheimer's disease.

\section{Case report}

A 78 year old white woman began to have subtle memory and personality changes in 1987. She complained of dyspnoea in December 1987, and $x$ ray and CT scan of the chest at another hospital suggested two left lobe nodules. Bronchoscopy and biopsy were negative. In April 1989, repeat CT scan revealed hilar adenopathy, but bronchoscopy was again negative. In June 1989, she was evaluated for unsteady gait and numbness in her hands. A CT scan of the chest showed improvement. CSF was normal except for a protein of $122 \mathrm{mg} / \mathrm{dl}$. CT and MRI scans of the head were unremarkable except for mild cortical atrophy. She became confused and incontinent and was transferred to the University of Virginia Health Sciences Centre.

On admission the patient was alert but confused, oriented only to person. She had severe memory and cognitive impairment. Cranial nerve examination was normal. Strength was $4 / 5$ in all muscle groups with mild hypotonia. There was marked dysmetria. Sensory examination was inconsistent. Deep tendon reflexes were absent with bilateral Babinski responses. Chest $x$ ray showed no masses. CSF was sterile with protein $70 \mathrm{mg} / \mathrm{dl}$ and 6 WBCs. EEG revealed bihemispheric slowing. An MRI scan of the head (fig 1) showed increased signal intensity involving the medial temporal lobes on T2weighted images. The patient was treated

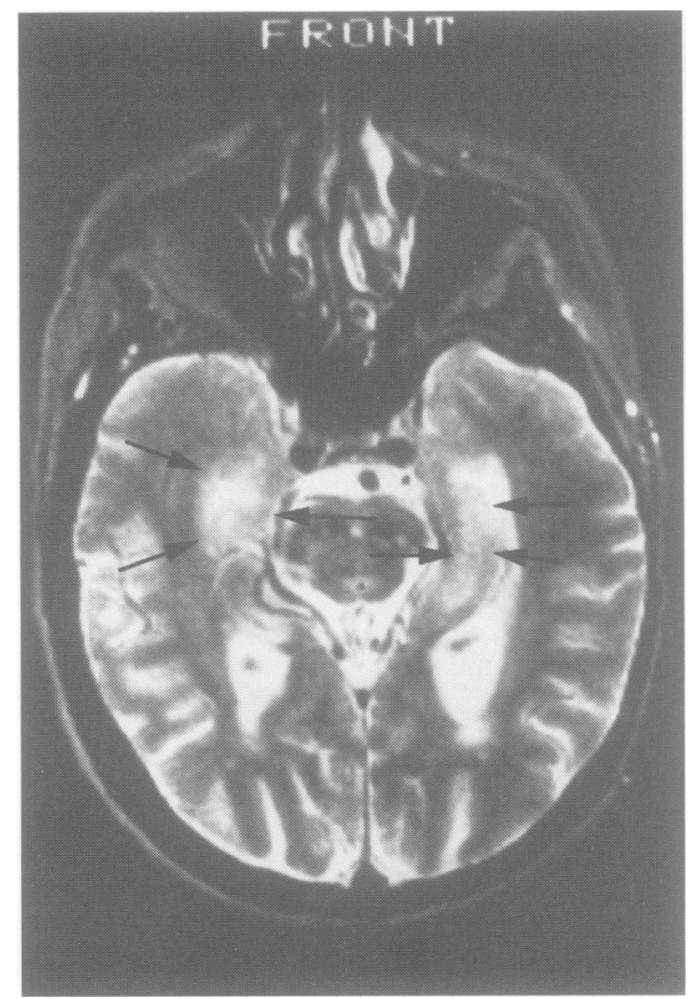

Figure 1 Axial T2-weighted (2500/90) MRI demonstrating diffuse increased signal intensity in the medial portions of both temporal lobes (arrows). 


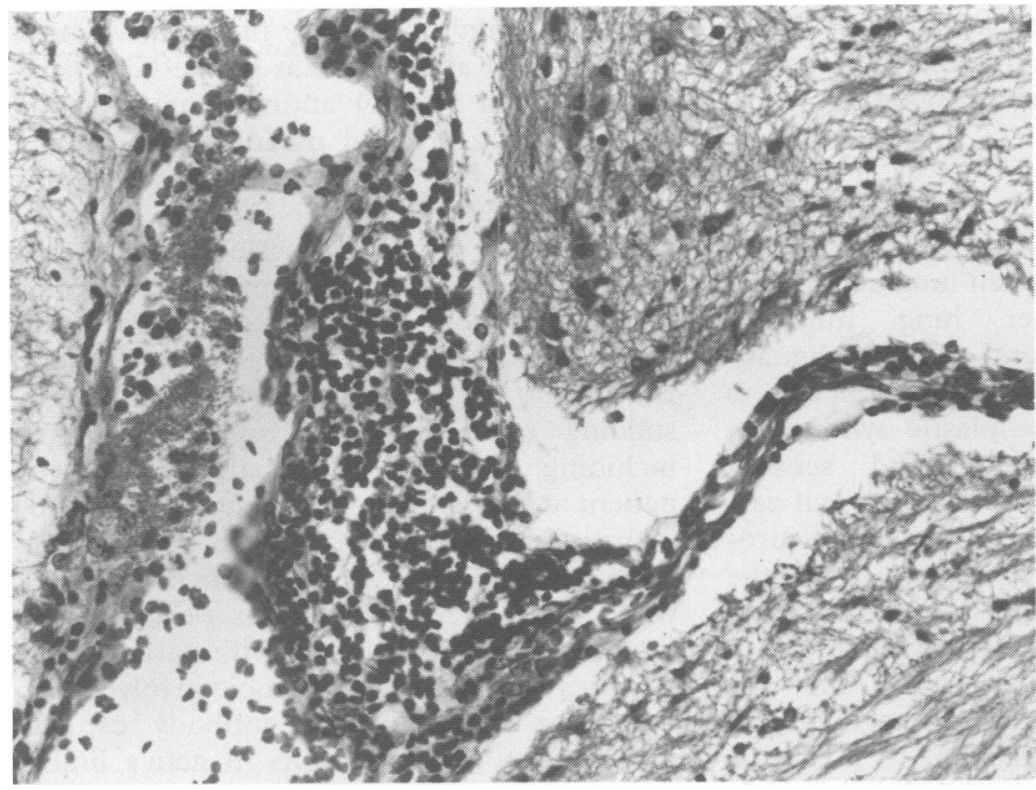

Figure 2 Section of the amygdala demonstrating perivascular lymphocytic infiltrate within the meninges (hematoxylin and eosin, $\times 250$ ).

empirically with acyclovir. A brain biopsy of the right temporal lobe showed perivascular lymphocytic infiltrates in the leptomeninges. She deteriorated rapidly, became comatose and died on 22 August 1989.

\section{PATHOLOGY}

Necropsy revealed pulmonary emphysema with recent pulmonary emboli. A $2 \times 2.5 \mathrm{~cm}$ small-cell carcinoma of the lung was present in the left hilum. A $5 \times 9 \mathrm{~cm}$ adenocarcinoma of the colon was found.

The brain weighed 1290 grams and appeared grossly normal except for post surgical changes over the right temporal lobe. Microscopic examination revealed chronic perivascular inflammatory changes in the meninges (fig 2) and encephalitis with lymphocytic infiltrates of the medial temporal lobes. There was neuronal loss and gliosis in the olivary complex and ganglioradiculitis at multiple levels. There were recent small infarcts in the right amygdala, pontine tegmentum and left lateral basis pontis and old lacunar infarcts in the right globus pallidus. On Bielschowsky silver staining there were moderate numbers of neuritic plaques, greater than 15 per $\mathrm{mm}^{2}$ in all areas of the neocortex, amygdaloid bodies and hippocampal formations, meeting neuropathological criteria for Alzheimer's disease. ${ }^{6}$ There were numerous neurofibrillary tangles and granulovacuolar degeneration in the hippocampi and modest numbers of cortical neurofibrillary tangles.

IMMUNOFLUORESCENCE

Indirect immunofluorescence was carried out with the patient's serum as described previously. ${ }^{7}$ Serum was titrated against normal human cerebellum using FITC-conjugated goat anti-human IgG as secondary. Screening produced intense, finely granular nuclear and cytoplasmic staining of all neurons (fig 3 ) and no other cells in a type II pattern ${ }^{8}$ (anti- $\mathrm{Hu}^{4}$ ). The endpoint titration was 1:1,280. Normal

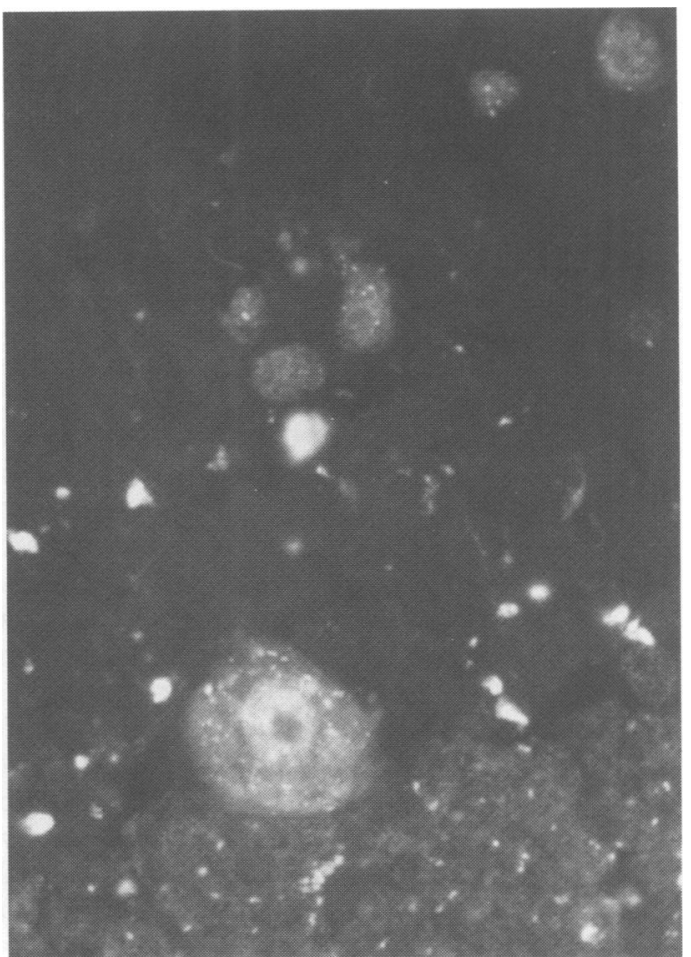

Figure 3 Indirect immunofluorescence of normal human cerebellum incubated with 1:80 dilution of patient serum followed by FITC-conjugated anti-human IgG. There is intense, granular labelling of nuclei and cytoplasm of all neurons typical of type II (anti-Hu) staining $(\times 475)$.

serum controls produced no staining.

Direct immunofluorescence was performed on frozen sections of the patient's necropsy tissue as described previously. ${ }^{9}$ Immunostaining produced moderate to intense cytoplasmic and nuclear labelling of most remaining neurons in temporal cortex indicating bound IgG (fig 4). Neurons in the cerebellum, brainstem and dorsal root ganglia

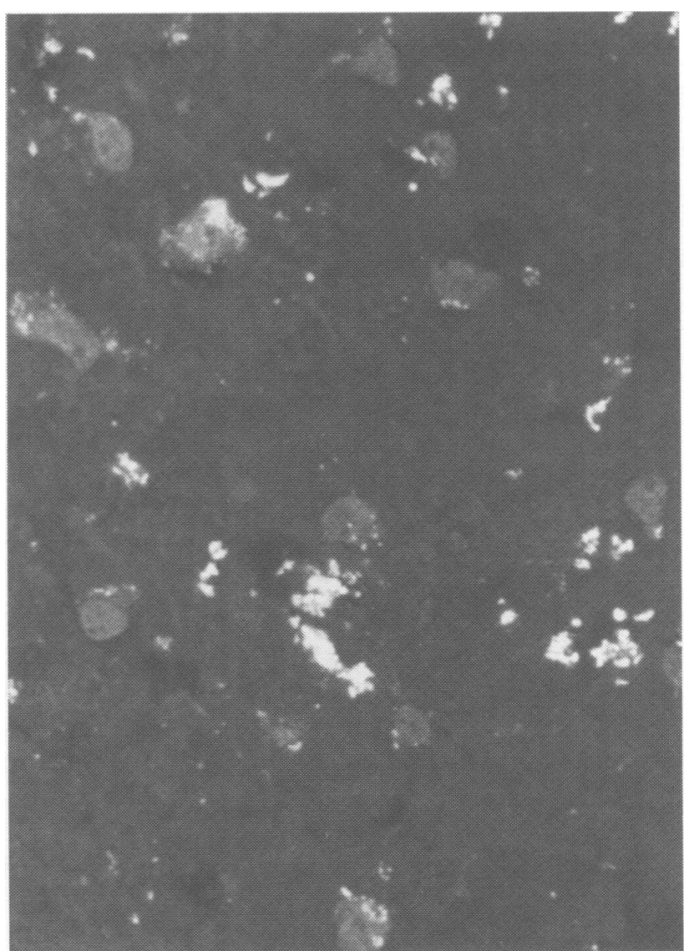

Figure 4 Direct immunofluorescence of patient's temporal cortex indicating bound IgG in most but not all neurons and no other cells $(\times 320)$ 
were also labelled but non-neuronal cell types were not stained. Direct immunofluorescence of normal brain produced no staining.

\section{Discussion}

Limbic encephalitis is a rare complication of cancer, most often small-cell lung carcinoma, but occasionally other lung tumours, Hodgkin's lymphoma, and other malignancies. ${ }^{3}$ Limbic encephalitis is one manifestation of a spectrum of paraneoplastic syndromes, including encephalomyelitis and sensory neuronopathy, associated with small-cell carcinoma. This group of paraneoplastic neurological syndromes is distinguished by the occurrence of circulating antineuronal antibodies which label nuclei and cytoplasm in a pan-neuronal (type II) pattern ${ }^{8}$ and are directed against neuronal antigens of 35-40 $\mathrm{Kd}$ molecular weight (anti-Hu). ${ }^{5}$

Paraneoplastic limbic encephalitis is usually characterised by subacute dementia. Alzheimer's disease, the most common cause of dementia, also has a predilection for limbic structures and usually presents with the insidious onset of similar symptoms. As illustrated by this patient, the gradual progression of a degenerative dementia can obscure the onset of limbic encephalitis. Subacute behavioural changes are not uncommon in patients with Alzheimer's disease, frequently due to medication effects, systemic illnesses or changes in environment. Memory impairment in both disorders compromises the patient's ability to give an accurate history. Routine laboratory and radiographic features usually show little or no abnormalities. Thus the initial clinical features of the onset of limbic encephalitis may be ignored or attributed to other causes.

Most cases of paraneoplastic limbic encephalitis were recorded before the advent of CT and MRI. The few reported cases indicate CT is insensitive in this condition as it is unremarkable or may show atrophy, ${ }^{1011}$ although contrast enhancement may be seen. ${ }^{12}$ MRI may show increased signal intensity on T2-weighted images in affected portions of the temporal lobes ${ }^{10113}$ (fig 1). Our report correlates these MRI findings in necropsy confirmed limbic encephalitis with demonstrated antineuronal antibodies. Late in the course of limbic encephalitis MRI scans may show evidence of temporal lobe atrophy, such as parenchymal volume loss and dilatation of the temporal horns. ${ }^{1012}$

Routine laboratory studies are usually of little help in the diagnosis of limbic encephalitis. ${ }^{3}$ CSF protein may be elevated with occasional white cells. In contrast, the detection of anti-neuronal antibodies is highly specific for a paraneoplastic neurological disorder. ${ }^{5}$ Indirect immunofluorescence demonstrated high serum titres of antineuronal antibody in a type II pattern typical for both encephalomyelitis and ganglioradiculitis associated with small-cell carcinoma of the lung. The specificity and noninvasiveness of indirect immunostaining emphasise the importance of early consideration of limbic encephalitis.
Direct immunostaining of neuron-bound antibodies in affected areas of the CNS suggests a direct role for antineuronal antibody in the pathogenesis of paraneoplastic limbic encephalitis, but few such clear associations have been reported in the literature. Several authors ${ }^{\text {14-18 }}$ have described neuronal immunostaining in patients with paraneoplastic disorders, but without precise correlation with clinical symptoms and pathology. Brashear et al demonstrated direct immunostaining of neurons in multiple regions, including temporal lobe structures in a patient with paraneoplastic encephalomyeloneuritis and prominent limbic encephalitis. Dalmau et $a l^{18}$ eluted antineuronal antibody with anti-Hu reactivity from post mortem brain tissue of five patients with paraneoplastic encephalomyelitis and limbic involvement. Our case confirms that antibody can be detected on target neurons in active limbic encephalitis and shows the utility of obtaining frozen tissue.

This report shows the extent to which cognitive impairment due to a progressive neurodegenerative disorder, such as Alzheimer's disease, can obscure the onset of a subacute dementia, in this case due to limbic encephalitis. Most laboratory tests do not narrow the differential diagnosis, however, MRI may demonstrate typical involvement of limbic structures. Antineuronal antibody screening can be diagnostic, indicating the presence of occult carcinoma responsible for the paraneoplastic disorder.

This work was supported in part by NIH grants AG 00407 and T32NS7236. We thank Dr P Colopy for referring the patient, Ms P Keeney for excellent technical assistance, and Ms R Powell for help in preparing the manuscript.

1 Brierly JB, Corsellis JAN, Hierons R, Nevin S. Subacute encephalitis of late adult life mainly affecting the limbic areas. Brain 1960;83:357-68.

2 Corsellis JA, Goldberg GJ, Norton AR. Limbic encephalitis and its association with carcinoma. Brain 1968;91:481-96.

3 Henson RA, Urich H. Cancer and the nervous system: the neurological manifestations of systemic malignant disease. Boston:Blackwell, 1982

4 Graus F, Elkon KB, Lloberes P, et al. Neuronal antinuclear antibody (anti-Hu) in paraneoplastic encephalomyelitis simulating acute polyneuritis. Acta Neurol Scand 1987;75:249-52.

5 Anderson MB, Rosenblum MK, Graus F, Wiley RG, Posner JB. Autoantibodies in paraneoplastic syndromes associated with small-cell lung cancer. Neurology 1988; 38:1391-8.

6 Khachaturian Z. Diagnosis of Alzheimer's disease. Arch Neurol 1985;42:1097-1105.

7 Greenlee JE, Brashear HR. Antibodies to cerebella Purkinje cells in patients with paraneoplastic cerebella degeneration and ovarian carcinoma. Ann Neuro 1983;14:609-13.

8 Jaeckle KA, Greenlee JE. Immunohistochemical pattern of antibody response in paraneoplastic neurological syndromes correlate with specific syndromes and with tumor types (abstract). Ann Neurol 1988;24:121.

9 Brashear HR, Caccamo DV, Heck A, Keeney PM. Localization of antibody in the nervous system of patient with paraneoplastic encephalomyelitis. Neurology 1991;41:1583-7.

10 Dirr LY, Elster AD, Donofrio PD, Smith M. Evolution of brain MRI abnormalities in limbic encephalitis. Neurology 1990;40:1304-6.

11 Lacomis D, Koshbin S, Schick RM. MR imaging of paraneoplastic limbic encephalitis. $\mathcal{F}$ Comput Assist Tomogr 1990;14:1 15-7.

12 Burton GV, Bullard W, Walther PJ, Burger PC Paraneoplastic limbic encephalopathy with testicular carcinoma. A reversible neurologic syndrome. Cancer 1988;62:2248-51. 
13 Kohler J, Hufschmidt A, Hermle L, Volk B, Lücking CH. Limbic encephalitis: two cases. $f$ Neuroimmunol 1988;20:177-8.

14 Graus F, Elkon KB, Cordon-Cardo C, Posner JB Sensory neuropathy and small-cell lung cancer: antineuronal antibody that also reacts with the tumor. $A m \mathcal{F}$ neuronal antibody that

15 Anderson NE, Budde-Steffen C, Wiley RG, et al. A variant of the anti-Purkinje cell antibody in a patient with paraneoplastic cerebellar degeneration. Neurology 1988;38:1018-26.

16 Graus F, Ribalta T, Campo E, Monforte R, Urbano A,
Rozman C. Immunohistochemical analysis of the immune reaction in the nervous system in paraneoplastic encephalomyelitis. Neurology 1990;40:219-22.

17 Grisold W, Drlicek M, Popp W, Jellinger K Antineuronal antibodies in small-cell lung carcinoma: a significance for paraneoplastic syndromes? Acta Neuropath for paraneoplastic

18 Dalmau J, Furneaux HM, Rosenblum MK, Graus F, Posner JB. Detection of the anti-Hu antibody in specific regions of the nervous system and tumor from patients with paraneoplastic encephalomyelitis/sensory neuronopathy. Neurology 1991;41:1757-64. 